\title{
MODEL-BASED DESIGN OF AM COMPONENTS TO ENABLE DECENTRALIZED DIGITAL MANUFACTURING SYSTEMS
}

\author{
Borgue, Olivia (1); \\ Stavridis, John $(2,3)$; \\ Vannucci, Tomas (4); \\ Stavropoulos, Panagiotis (5); \\ Bikas, Harry (5); \\ Di Falco, Rosa (2,3,); \\ Nyborg, Lars (1) \\ 1: Chalmers University of Technology; \\ 2: Prima Industries SpA; \\ 3: Politecnico di Torino; \\ 4: RISE Research Institutes of Sweden; \\ 5: University of Patras
}

\begin{abstract}
Additive manufacturing (AM) is a versatile technology that could add flexibility in manufacturing processes, whether implemented alone or along other technologies. This technology enables ondemand production and decentralized production networks, as production facilities can be located around the world to manufacture products closer to the final consumer (decentralized manufacturing). However, the wide adoption of additive manufacturing technologies is hindered by the lack of experience on its implementation, the lack of repeatability among different manufacturers and a lack of integrated production systems. The later, hinders the traceability and quality assurance of printed components and limits the understanding and data generation of the AM processes and parameters. In this article, a design strategy is proposed to integrate the different phases of the development process into a model-based design platform for decentralized manufacturing. This platform is aimed at facilitating data traceability and product repeatability among different AM machines. The strategy is illustrated with a case study where a car steering knuckle is manufactured in three different facilities in Sweden and Italy.
\end{abstract}

Keywords: Additive Manufacturing, Systems Engineering (SE), 3D printing

\section{Contact:}

Borgue, Olivia

Chalmers University of Technology

Industrial and Material Sciences

Sweden

borgue@chalmers.se

Cite this article: Borgue, O., Stavridis, J., Vannucci, T., Stavropoulos, P., Bikas, H., Di Falco, R., Nyborg, L. (2021) 'Model-Based Design of AM Components to Enable Decentralized Digital Manufacturing Systems', in Proceedings of the International Conference on Engineering Design (ICED21), Gothenburg, Sweden, 16-20 August 2021. DOI:10.1017/ pds.2021.474 


\section{INTRODUCTION}

Manufacturers are looking for reducing cost in their production lines, while increasing speed and ensuring repeatability. In this context, literature (Rayna et al., 2016) suggests that additive manufacturing (AM) could reduce lead time costs reducing supply chain stages, possible through manufacturing products with fewer components, and reducing transportation, packaging and warehousing. In this context, additive manufacturing technologies can increase the market strength and responsiveness, while providing functionally enhanced products with short lead times (Naghshineh and Cavalho, 2020). These technologies could also add flexibility to the supply chain (Naghshineh and Cavalho, 2020), manufacturing products on demand and locating manufacturing facilities closer to the customers (distributed, decentralized manufacturing). Bogers et al. (2016) and Savolainen and Collan (2020) refer to these supply chains as being "glocalized", as they possess a local self-sufficiency with a global implementation.

However, additive manufacturing technologies are yet not widely implemented. The lack of AM adoption is due to: (1) Lack of experience regarding AM implementation, (2) Lack of manufacturing repeatability, as the product outcome is dependent on the machine, machine parameters, materials, and geometry, and (3) Lack of integrated production systems: The "glocalized" approach for distributed manufacturing is not yet supported by current technological capabilities (Savolainen and Collan, 2020; Naghshineh and Cavalho, 2020).

Current integrated production systems for AM have limitations regarding the use of the data they can capture, analyse and process (Bonham et al., 2020). Data capture, analysis and processing are generally limited to isolated steps in the supply chain, such as simulation or manufacturing, which leads to the creation of data silos where the data collected is never shared or used to complement other steps of the manufacturing process. This data isolation hinders the establishment of an AM digital thread, traceability, quality assurance and the implementation of decentralized manufacturing strategies (Bonnard et al., 2018; Bonham et al., 2020). As a result, decentralized manufacturing systems might fabricate components that are not repeatable or that are too expensive or take too long time to be manufactured in certain locations.

There is a need for gathering and analysing the data from every AM process step and AM manufacturer, and use it on a unified digital platform to enable the design of cost and time efficient AM products, for decentralized manufacturing approaches. For this reason, this article intends to solve the research question:

RQ: How can a unified digital platform support multidisciplinary data utilization to design cost and time-efficient AM products in the context of decentralized manufacturing strategies?

The aim of this article is to provide a model-based design method for utilizing data from various AM manufacturers during the whole lifecycle to enable the design of cost and time-efficient AM products in the context of decentralized manufacturing integrated production systems. The design method is illustrated with a case study featuring the redesign for AM of a car steering knuckle. The knuckle is meant to be manufactured in three different locations in Sweden and Italy.

\section{BACKGROUND}

Literature suggests (Savolainen and Collan, 2020; Naghshineh and Cavalho, 2020) a need for strategies to manage AM data across its lifecycle to ensure repeatability in decentralized manufacturing systems. Such strategies should be flexible enough to allow for new AM partners (designers, suppliers, etc.) and lead to an understanding of the complex interaction of material, processes, and component geometry, leading to their optimization (Miles et al., 2016; Bonham et al., 2020).

Authors such as Bonnard et al. (2018), Bonham et al. (2020) or Liu et al. (2020), proposed strategies for designing and integrating data capture and analysis models for AM technologies. They proposed comprehensive platforms that enable multidisciplinary data analysis, tracking and visualization with the objective of improving production. Moreover, Bonham et al. (2020) focused their work on decentralized manufacturing systems, extending their data platform to fit several manufacturers. However, these strategies do not propose the use of the collected data to facilitate design decisions and optimize component geometry. 
The same concern can be raised about software platforms for AM data capture and manage in general. The authors Miles et al., (2016), review a catalogue of open source and commercial software platforms for AM that capture and manage data from the whole component lifecycle. These platforms are useful extensions of existing data management technologies and work mostly as visualization tools (Miles et al., 2016); however, they do not propose strategies to use the data for design or process improvements purposes.

To use data from the whole product lifecycle and improve component and processes design, the data must be available in design phases of the product development process. In the case of innovative designs, or when implementing new technologies, these data are obtained through early, long and expensive test campaigns (Brice, 2011). In the case of decentralized manufacturing systems, as the number of manufacturers increase, an increase on the cost of the testing phases is expected.

To reduce the cost of test campaigns, authors such as Rupal et al. (2018) or Borgue et al. (2019) proposed the use of functional and non-functional requirements (functions and constraints) modelling techniques to identify design, manufacturing process and quality uncertainties during design phases and design tailor made test artefacts to collect specific required information. These strategies succeed at modelling the available information in a comprehensible platform and implementing that information to propose cost-effective test strategies to optimize a component design and ensure product quality. Moreover, function modelling strategies in general, act as comprehensive tools for information documentation and communication among stakeholders (Eisenbart et al., 2017; Müller et al., 2020). Nevertheless, the work by Rupal et al. (2018) or Borgue et al. (2019) is focused on optimizing components manufactured in only one AM machine, therefore, do not propose strategies to address manufacturing repeatability in decentralized manufacturing scenarios.

The preceding review suggest a need for a design method to enable the implementation of lifecycle data during design phases to design cost and time-efficient AM products in the context of decentralized manufacturing integrated production systems.

\section{MATERIALS AND METHODS}

In this article, the results of a project held in the context of an advanced manufacturing demonstration project, are discussed. The project was held as an international collaboration between universities, research institutes and companies from Sweden and Italy, and had the objective of improving data traceability and quality control in the context of AM products.

In this context, this article presents the project efforts to develop a model method to anticipate production challenges and optimize production inputs for reducing lead times and costs in decentralized manufacturing scenarios.

The activities performed during the project feature a series of meetings and workshops attended by industrial practitioners from the participating organizations (Figure 1). The activities iterate technical meeting and design/analysis iterations to combine the insights of researchers and practitioners, reflecting on a design problematic and aiming to propose a solution from different points of views.

The first step was a proposition of a case study, a component to be redesign for AM and manufactured in three different locations in Sweden and Italy.

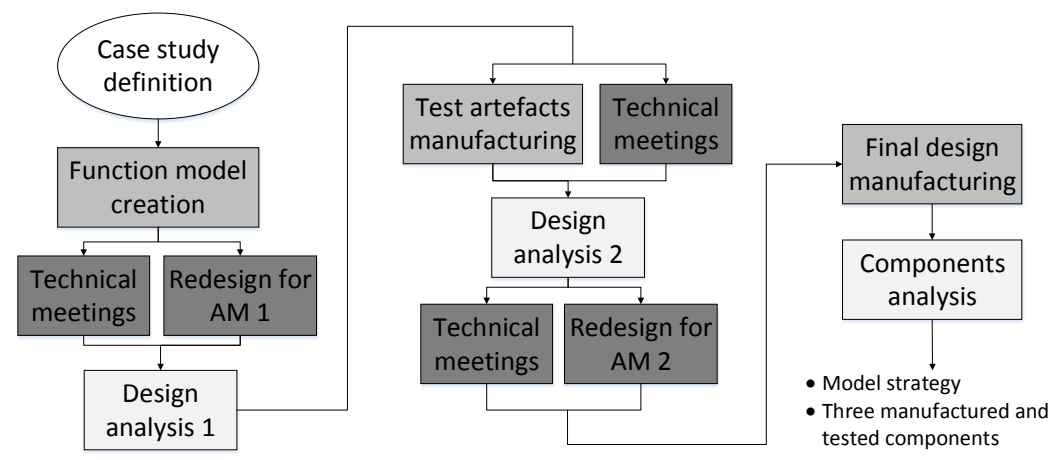

Figure 1. Design method followed, to reduce lead times and costs for decentralized manufacturing scenarios. 
Later, through meetings and functional analysis, a function model (FM) was built. Based on the FM, the first design iteration was performed, and the component was redesigned for AM. Parallel to the redesign process, a series of technical meetings were held with the representatives of the three participating manufacturing centres, to iterate the design regarding its functional and manufacturing requirement. The first design iteration enabled a round of design data analysis, where the design was preliminary evaluated regarding aspects such as weight, post processing time or material consumption. From the data analysis, a series of test artefacts were designed and build to gather specific data about AM machine capabilities at the three manufacturing locations. The obtained machine data was analysed to improve the final concepts design, which were then printed at the three locations and analysed. The output of these activities was three manufactured and analysed components and the model design method presented in this article.

\section{MODEL-BASED DESIGN METHOD FOR DECENTRALIZED MANUFACTURING}

The proposed model-based design method for decentralized AM is divided into five main steps, introduced in Figure 2.

1. Construction of a function model.

2. Component redesign for AM and design analysis

3. Test artefacts manufacturing and analysis

4. Design iteration and analysis

5. Design manufacturing

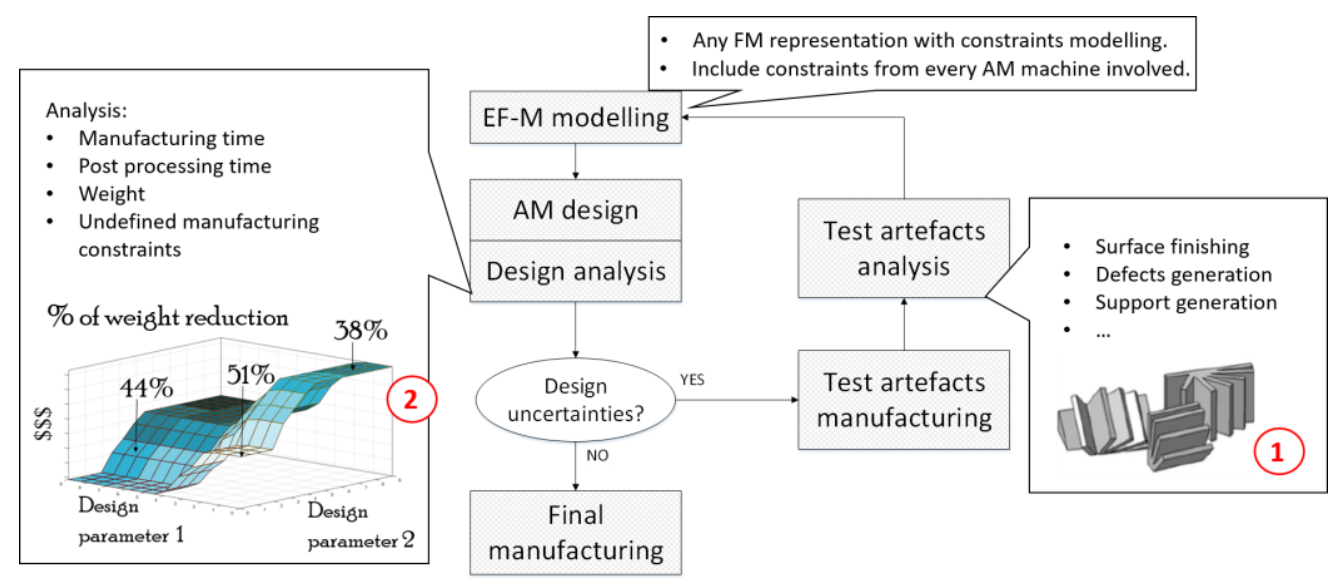

Figure 2. Design method flow diagram

\subsection{Step 1: EF-M model construction}

An analysis of the component functional and non-functional requirements is performed (Rupal et al., 2018), and they are then organized in a function model to facilitate understanding of the product architecture and store manufacturing information (Borgue et al., 2019). In this article, the function modelling technique "Enhanced Function-Means" (EF-M) (Claesson, 2006) was preferred, as it provides the possibility of modelling of constraints (non-functional requirements), which is crucial for this design method and manufacturing analysis (Müller et el., 2020), however other model strategies with similar capabilities could be used. In the EF-M technique, presented in Figure 3, the component geometry is analysed to find functional requirements (FRs) and their associated design specifications (DSs). Then, constraints (C) are identified and included in the model, associated with their applicable DS. For example, to satisfy the FR "transfer fluid", a flow connector is an appropriate DS. Flow requirements, such as pressure and temperature, are performance constraints that limit design parameters such as dimensions or materials. Moreover, manufacturing constraints (such as minimum wall thickness) limit the design as well.

It is expected to encounter discrepancies among the manufacturing constraints of the different machines. However, every manufacturing constraint must be included in the model.

After the EF-M model is built, the component is redesigned for AM. 


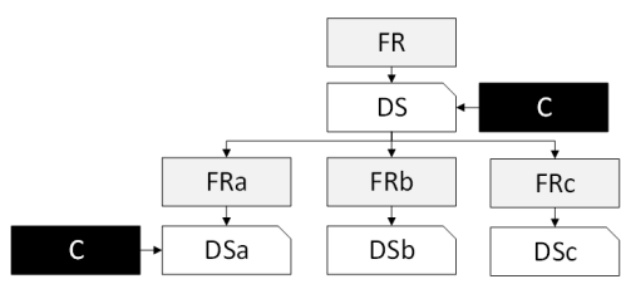

Figure 3. Enhanced function-means representation (Claesson, 2006)

\subsection{Step 2: Component redesign for $A M$ and design analysis}

In this Step, an AM design is developed from the EF-M model. During the first design iterations, design uncertainties are expected, and the design must be analysed to find them (Rupal et al., 2018). These uncertainties can be related to manufacturing, manufacturing constraints, quality, etc.

As manufacturing constraints are machine, parameters, material, and geometry dependent (Savolainen and Collan, 2020; Naghshineh and Cavalho, 2020), a further evaluation of the manufacturing constraints for each machine process might be necessary. The processes of AM design and design analysis are iterative, as results from the design analysis should be implemented to refine the AM design.

For example, when designing a component with an overhang, a 45 degrees overhang angle is recommended in literature for avoiding support structures. However, authors, such as Jiang et al. (2018), demonstrated that lower overhang angles without support structures can be manufacturable adjusting process parameters. Authors such as Rupal et al. (2018) and Borgue et al. (2019) have proposed strategies for design analysis based on systematic identification of uncertainties, to assess geometry manufacturability as well as machine capabilities.

Further design analysis can be performed through multidimensional trade-off analysis tools such as multidimensional plots, or parallel coordinates graphs (Berthold and Hall, 2003). The iterative design and analysis process aims at designing a component that can be manufactured in every AM facility of interest and that would provide the highest value (for further details see Step 4).

If after the design and analysis process, no design uncertainty is found, the component is ready for manufacturing. However, if design uncertainties are found, a series of test artefacts must be designed and manufactured to gather the missing data.

\subsection{Step 3: Test artefacts manufacturing and analysis}

In this Step, test artefacts are designed and manufactured to gather data about design uncertainties. There are different strategies to design test artefacts for AM. However, authors such as Rupal et al. (2018) and Borgue et al. (2019) proposed tailoring the test artefacts according to the component geometry and functionality. In Figure 2, (1) test artefacts used by Borgue et al (2019) for evaluating surface inclination vs. surface finishing, are presented. Test artefacts must be manufactured with the same conditions than the objective products regarding machine parameters, materials, and position on the building plate.

Test artefacts should then be analysed to obtain the required data. The results will be used to update the EF-M model and data in the trade-offs tools during design analysis,

Surface finishing can be improved through post-processing activities. A study about surface inclination angle and the resulting surface finishing, for example, can provide data about the relationship between surface inclination and post-processing time. This way, a designer could vary the design parameter "surface inclination" obtaining different values for the post-processing time.

\subsection{Step 4: Design iteration and analysis}

Once the EF-M model is updated, the AM component design can be iterated and analysed again. When more information is available, it is possible to refine the trade-off analysis. In Figure 2, (2) represents a multivariable plot where different combinations of values of the design parameters 1 and 2, yield different results regarding costs and percentage of weight reduction. The information to populate such graph can be obtained from test artefacts, evaluating the design parameters combinations and their influence of, for instance, post processing time, which impacts the total fabrication cost.

The sequence "AM design-analysis-test artefacts-redesign" should be applied to every design project, regardless of the number of AM machines involved. As the number of manufacturing facilities increases, a systematic modelling and analysis method gains importance. Figure 4 presents three design scenario when designing a component to be manufactured by two machines with different capabilities. In this example, a strategy could be to design a component to fit the machine with less restrictive manufacturing 
capabilities, Machine A in Figure 4, and increase post processing times for the less capable machine (B) (Figure 4.a). Another alternative (Figure 4.b) is to design a component to fit the machine with more restrictive capabilities (Machine B), ensuring manufacturing and reducing post processing time respect to the first option. However, a third option (Figure 4.c) adjusts the design to find a midground between both machines, looking to minimize post processing time of the whole manufacturing operation.

The example presented in Figure 4 is rather simple and systematic model strategies might not be necessary. However, more complex geometries require the implementation of data intensive multidisciplinary design analysis and optimization techniques, especially in scenarios where a larger number of machines and design parameters are involved.

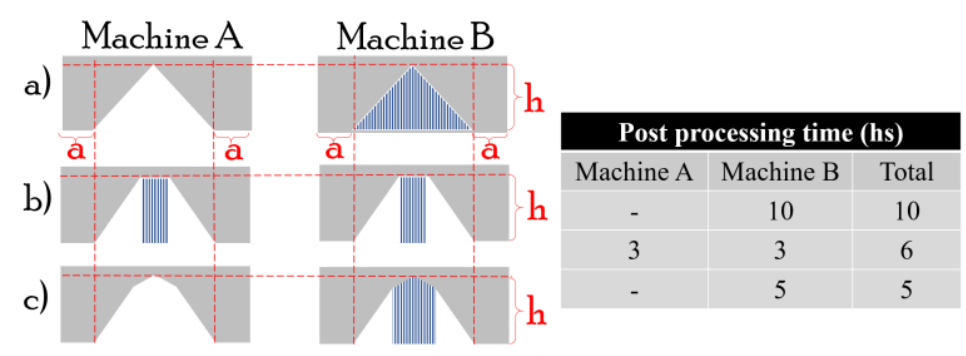

Figure 4. Example of three possible design scenarios for decentralized manufacturing

\subsection{Step 5: Design manufacturing}

When enough information has been gathered to shed light into every found design uncertainty, and the process of design iteration-design analysis settled with a component design, the component can be manufactured.

\section{REDESIGN OF A STEERING KNUCKLE FOR DECENTRALIZED ADDITIVE MANUFACTURING SCENARIOS}

The steering knuckle is a component in a car suspension system for connecting the steering components with the wheel. This component is usually machined from an Inconel block and must withstand the loads from the wheel and steering system, preserving wheel alignment and stability. The knuckle in this project is meant to be manufactured with powder-based AM technologies. In Figure 5, the steering knuckle geometry and EF-M model are presented. Some Cs were obtained from the Non-Functional Requirements (or constraints) list for the component. Other constraints, related with the manufacturing technology of choice were obtained from general AM design guidelines (Diegel et al., 2019) and machine datasheets. The design was iterated to achieve a preliminary reduction of support structures.

The objective of this case study is to design a steering knuckle to be manufactured at three different locations, two in Sweden and one in Italy, striving to reduce the total costs (manufacturing and postprocessing) and manufacturing time of the hole manufacturing endeavour to increase market strength and responsiveness, while providing a functionally enhanced product (Rayna et al., 2016; Naghshineh and Cavalho, 2020).

In this case, the manufacturing time comprehends AM machine operation and post-processing. The costs considered are those incurred from material consumption, AM machine operation and postprocessing. Post-processing activities include detaching the component from the building plate, removal of support structures and surface treatment. The AM machines considered in this study are (M1) Print Sharp 250, (M2) SLM Solution SLM125HL, and (M3) EOS M290.

Following the proposed methodology, the knuckle was designed for AM. For this purpose, a topology optimization analysis (Diegel, 2019) was performed on the maximal geometrical design space to obtain the most efficient material allocation. Later, respecting the constraints identified in the function model, the design was iterated to reduce the use of support structures.

However, as constraints are machine parameters, material, and geometry dependent (Savolainen and Collan, 2020), an evaluation of surface roughness and threshold angle for support structure for the three machines involved in the manufacturing process was performed. The purpose of these analysis is to refine the component dimensions, considering that when the surface is not smooth enough, a layer of material must be removed. Moreover, rough surfaces enable stress concentration and crack propagation. One strategy to increase robustness in components with rough surfaces, is to increase wall thicknesses. 


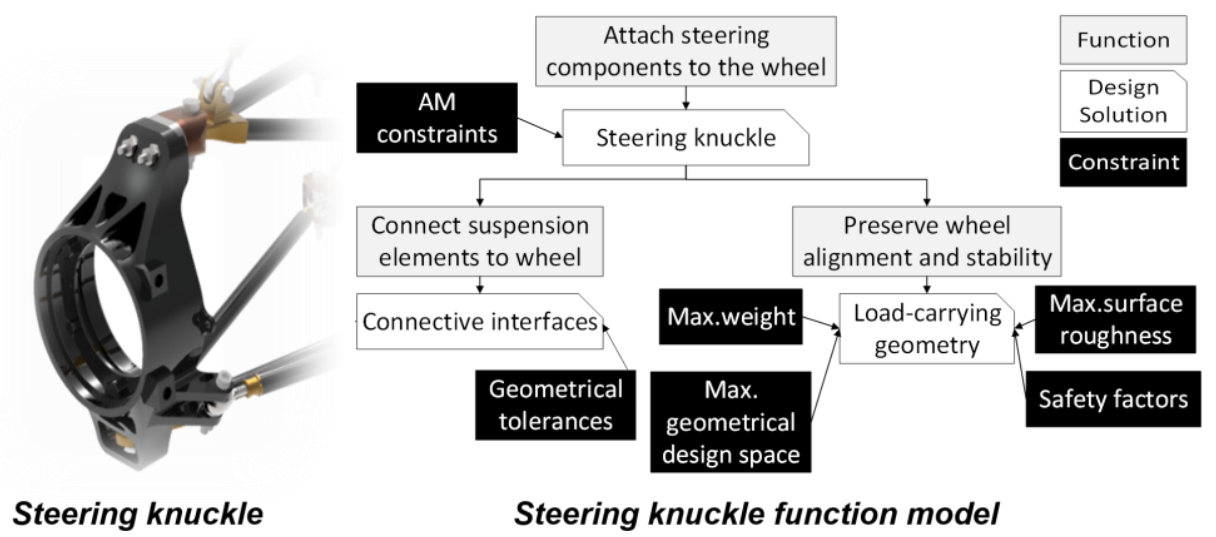

Figure 5. Steering knuckle (left), and knuckle function model (right).

In this context, the design parameters that were analysed are surface inclination, due to its incidence in surface roughness, and wall thickness. In Figure 6 (top), the preliminary knuckle design is presented, displaying a preliminary vertical printing direction. Four variable design parameters are presented:

1. Parameters $\alpha$ and $\tau$ : At the top of the knuckle, the surface inclination $\alpha$ can be modified, resulting in different surface roughness of up-facing surfaces; if, when $\alpha$ results in a rough surface, the wall thickness $\tau$ is drastically reduced, the component could end up in a critical failure situation.

2. Parameters $\beta$ and $\tau 2$ : At the bottom of the knuckle, the variation of angle $\beta$ results in different surface roughness in up- and down-facing surfaces. After a certain roughness threshold, material should be removed to improve the surface finishing. If the thickness $\tau 2$ is too thin, the component could face a critical failure.

Using data from literature (Diegel et al., 2019) and machine specifications, the above-mentioned parameters were varied to observe their impact in metrics such as weight, estimated manufacturing costs and time. These design variations were performed manually, which was proven to be extremely labour intensive. Examples of these analysis are found in Figure 6. From the parametric graphs in Figure 6, two areas of interest are identified for parameters combinations of $\alpha$ and $\tau$, and $\beta$ and $\tau 2$.
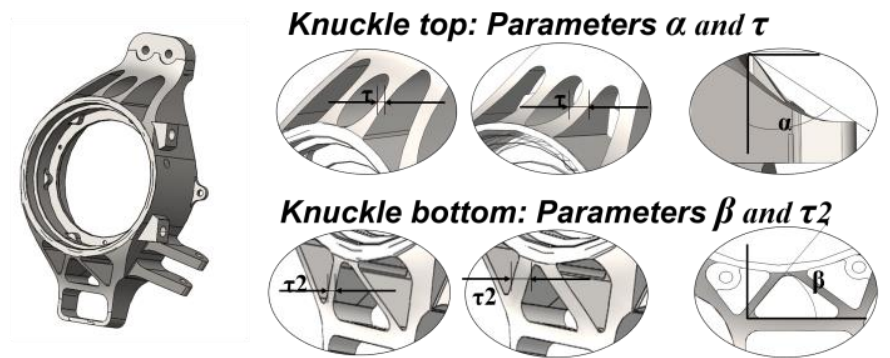

Preliminary parameters analysis:

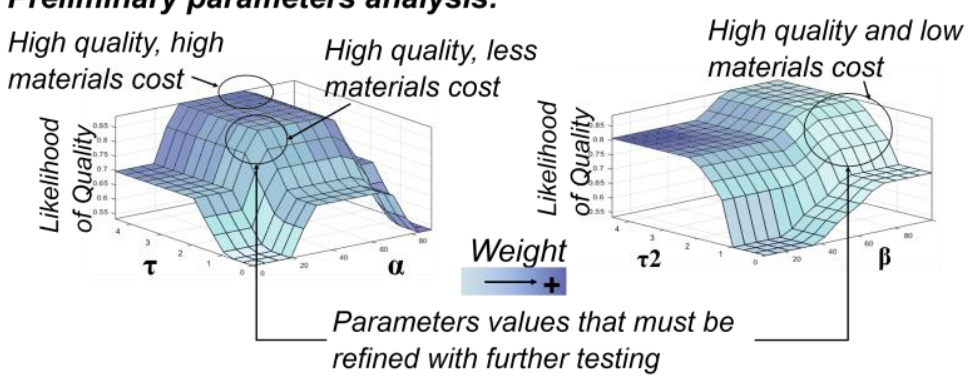

Figure 6. Parameters analysis evidence relevant design uncertainties to test further

Quality or structural robustness, related to surface roughness and wall-thickness, can also be analysed following the method suggested in this article. Forecasting quality during design phases to determine design parameters that require further testing is hindered by the lack of information that characterized early design phases. A convenient tool to perform such analysis is the implementation of Multi-Expert Multi- Criteria Decision Making (ME-MCDM) fuzzy approaches (Kaya et al., 2019), on experts' judgement, for modelling AM process uncertainties and their impact on product quality ("Likelihood of quality" in Figure 6). However, this analysis is the topic of a different study. 
For the three AM machines implemented in this project, the data available did not include a mapping of surface inclination angle and its incidence on surface roughness. This lack of data hinders the knuckle design refinement. Consequently, test artefacts (Figure 7, top left) were designed to collect the missing data and refine the parameters graphs on the areas of interest. The refined parameters graphs (Figure 7 top, 2 graphs per machine) are then used to improve the detail level of the AM constraints in the function model, as suggested by Borgue et al. (2019), which serves as visualization, documentation, and communication platform.

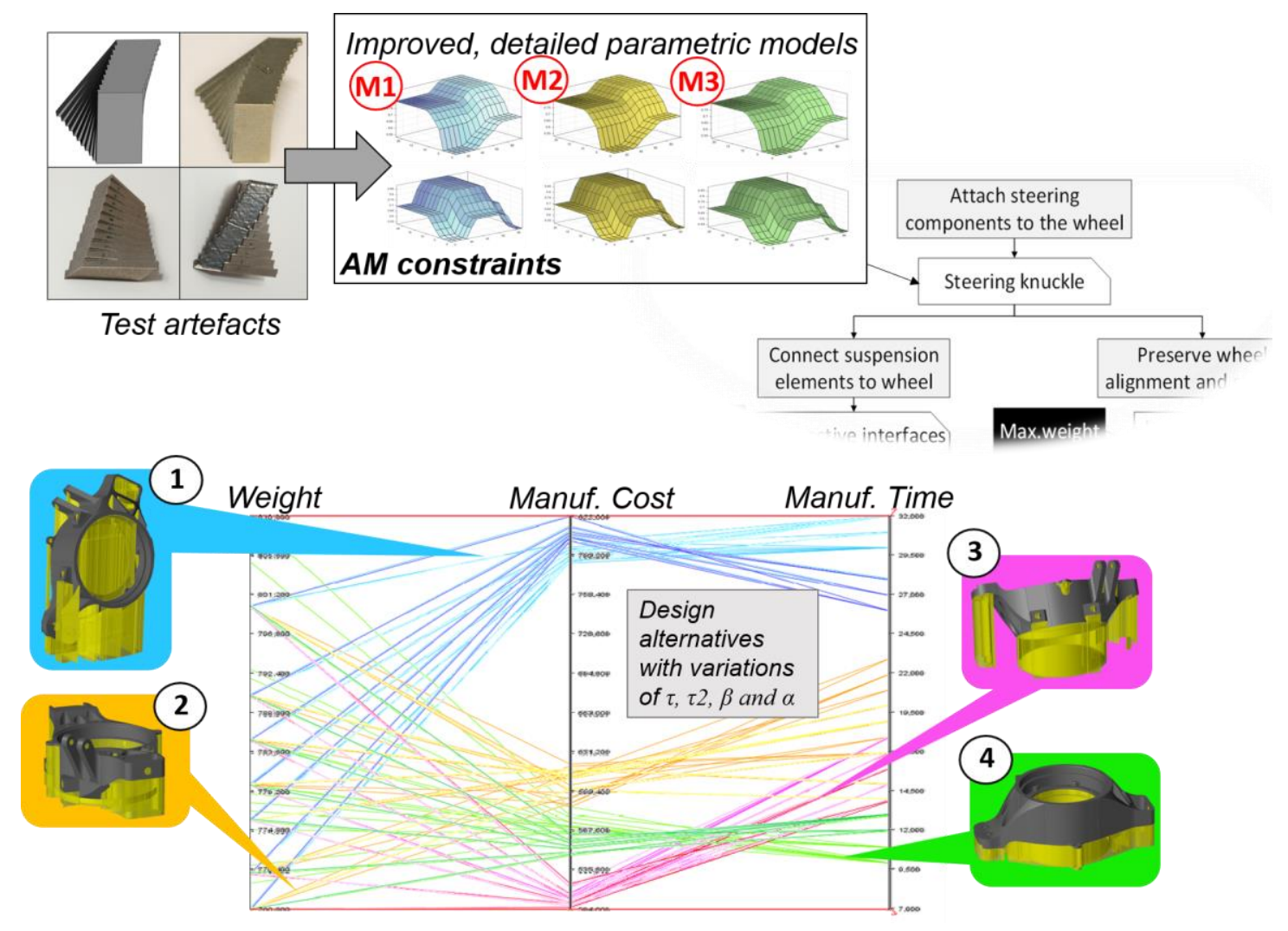

Figure 7. Information documentation and design decision making processes

With the new data gathered and documented, the knuckle design can be refined, choosing the parameters combinations that would provide the best results regarding of weight, manufacturing cost and manufacturing time (machine set up and prep time + printing time + post-processing time). Manufacturing costs are a function of the manufacturing time, and its respective resources. The "best results" are subjective and project and business context dependent. In Figure 7 bottom, a parallel coordinates graph is presented. In the graph, seven different design alternatives are presented, evaluated in terms of weight, manufacturing cost and manufacturing time. As printing orientation impacts weight and manufacturing costs and time, design decisions should be made concurrently with printing orientation decisions. On the parallel coordinates graph on Figure 7, the design alternatives are grouped according to 4 different printing orientations (blue, orange, pink and green). For each printing orientation, the seven design alternatives are evaluated for each of the three machines and presented in the graph as shades of the orientation colour (some of them imperceptible in the graph). Printing directions 3 and 4 are those presenting the lowest manufacturing costs and times, each of them favouring one of those metrics (printing alternative 3 has the lowest manufacturing costs and alternative 4 , the lowest manufacturing times). In this study, printing alternative 4 was preferred, as alternative 3 requires post-processing activities on the curved (downward) surfaces of the knuckle. These surfaces are considered critical and the effect that post-processing activities can have on the mechanical properties of those surfaces is unknown.

In Figure 8, a parallel coordinates graph focused on printing direction 4, is presented. Each colour represents a design alternative (7 design alternatives) obtained through variations of the parameters $\alpha$, $\beta, \tau$ and $\tau 2$. The results from each machine are also displayed (there are three lines for each colour). The selection of the final parameters' combination is design and business context dependent. In this 
study, the preferred design is the one with the lowest manufacturing costs. In other design situation, other design alternative be preferred. For passenger's cars, cost might be the most important metric, for formula car knuckles, however, weight is the most relevant metric.

After selecting the preferred design alternative and studying its manufacturing metrics for the three AM machines, the steering knuckle was printed in the three locations.
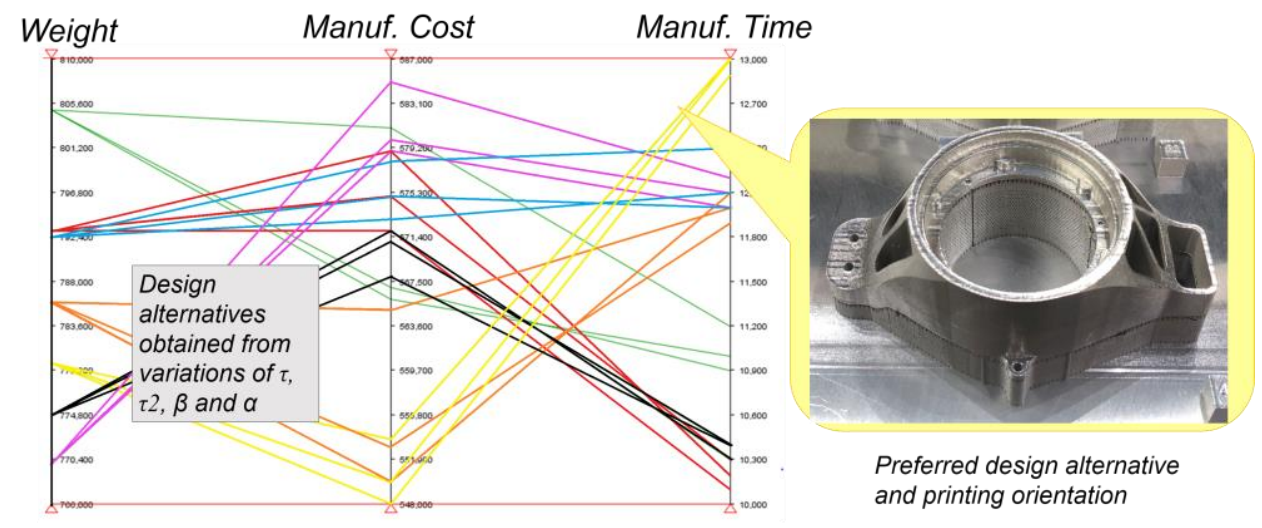

Figure 8. Performance of design alternatives obtained through parameters variations.

\section{DISCUSSION AND CONCLUSIONS}

In this article, a model-based design method based on lifecycle data utilization for decentralized manufacturing scenarios, was presented. The proposed method was elaborated from the results of an advanced manufacturing demonstrator project, held in an international context. In concordance with literature (Bonham et al., 2020; Savolainen and Collan, 2020; Naghshineh and Cavalho, 2020), one of the main concerns addressed in the project was manufacturing repeatability among manufacturers.

The proposed method addresses repeatability through the implementation of iterative steps of design, design analysis and testing. Early manufacturing of tailored test artefacts (Rupal et al., 2018; Borgue et al., 2019), reduces the cost of the test campaigns and contributes to manufacturing repeatability (Bonnard et al., 2018; Bonham et al., 2020).

A detailed design analysis must be performed to determine design uncertainties and the design parameters that require further testing. Such is the case of the parameters $\alpha$ and $\tau$, and $\beta$ and $\tau 2$ in this study. As the purpose of this article is to showcase the design method, only the test artefacts to establish the relationship between surface inclination and surface roughness are presented. However, in the design of a steering knuckle, further studies should be conducted to study how the different values for wall thickness and surface roughness affect the component structural robustness and quality in general.

The design and analysis activities were performed manually, which was proven to be inefficient and would add additional development costs in a real case scenario. Even analysing the interaction of just four parameters, the designer juggles with at least four different types of software: (1) Function model platform to document design information, (2) CAD software for geometrical design, (3) Analysis software for parameters analysis, (4) AM machine software for including manufacturing details into the analysis. As the number of manufacturers increase, the design analysis phases become longer. These findings are aligned with the results presented by Bonham et al. (2020).

For rendering the whole operation feasible and cost-efficient, there is a need for developing an integrated design platform. This platform would be enabled by a digital thread, able to easily retrieve information from a product lifecycle and perform automatic (at least to some degree) design and design analysis.

As proposed by Rupal et al. (2018) and Borgue et al. (2019), in the presented method tailored test artefacts are utilized to perform parameters design analysis. The novelty of this study lays on the introduction of several AM manufactures and the goal of designing a component that better serves the system as a hole, in terms of costs, time, and performance.

As observed in the case study, during the process of design analysis design, alternatives can be evaluated according to various design and business contexts. For example, focus on weight reduction on a small-batch production of knuckles for formula cars, but focus on manufacturing costs for largebatch productions of passenger cars. 
The proposed method is flexible and enables the introduction of new AM partners, as deemed necessary by authors such as Bonham et al. (2020). Moreover, it supports the evaluation of multidisciplinary requirements, storing and conveying data through a function model. In fact, some of the surface roughness data needed for this study, had been already obtained for previous projects, but due to lack of documentation, the measurements had to be performed again. The function model also serves as a boundary object and communication tool among the different analysis models and design teams.

To conclude, this method does not replace existent data management tools, but it complements them using lifecycle data for design improvement. Furthermore, this method was applied to a single case study, further studies must be performed to establish its generalizability and validity.

\section{ACKNOWLEDGEMENTS}

The authors gratefully acknowledge the financial support from the EIT (European Institute of Innovation and Technology) Manufacturing and the companies participating on the DIGIQUAM project that made possible the data collection needed for this study.

\section{REFERENCES}

Berthold, M. R., and Hall, L. O. (2003) "Visualizing fuzzy points in parallel coordinates,” IEEE Transactions on Fuzzy Systems, 11(3), pp. 369-374, https://doi.org/10.1109/TFUZZ.2003.812696.

Bogers M., R. Hadar, A. Bilberg. (2016) Additive manufacturing for consumer-centric business models: implications for supply chains in consumer goods manufacturing, Technol. Forecast. Soc. Change 102 pp. 225-239.

Bonham, E., McMaster, K., Thomson, E., Panarotto, M., Müller, J. R., Isaksson, O., \& Johansson, E. (2020). Designing and Integrating a Digital Thread System for Customized Additive Manufacturing in MultiPartner Kayak Production. Systems, 8(4), 43.

Bonnard, R., Hascoët, J. Y., Mognol, P., \& Stroud, I. (2018). STEP-NC digital thread for additive manufacturing: data model, implementation and validation. International Journal of Computer Integrated Manufacturing, 31(11), 1141-1160.

Borgue, O., Müller, J., Leicht, A., Panarotto, M., \& Isaksson, O. (2019). Constraint Replacement-Based Design for Additive Manufacturing of Satellite Components: Ensuring Design Manufacturability through Tailored Test Artefacts. Aerospace, 6(11), 124.

Brice, C. A. (2011). Unintended consequences: How qualification constrains innovation. In Proceedings of the 1st World Congress on Integrated Computational Materials Engineering (p. 241). John Wiley \& Sons.

Claesson, A. A Configurable Component Framework Supporting Platform-Based Product Development. Ph.D. Thesis, Chalmers University of Technology, Gothenburg, Sweden, 2006.

Diegel, O., Nordin, A. and Motte, D. (2019), A Practical Guide to Design for Additive Manufacturing, Springer, Singapore. https://doi.org/10.1007/978-981-13-8281-9 .

Eisenbart, B.; Gericke, K.; Blessing, L.T. (2007) Taking a look at the utilisation of function models in interdisciplinary design: Insights from ten engineering companies. Res. Eng. Des. 28, 299-331.

Jiang, J.; Stringer, J.; Xu, X.; Zhong, R.Y. Investigation of printable threshold overhang angle in extrusion-based additive manufacturing for reducing support waste. Int. J. Comput. Integr. Manuf. 2018, 31, 961969,https://dx.doi.org/10.1080/0951192X.2018.1466398.

Kaya, İ., Çolak, M., \& Terzi, F. (2019). A comprehensive review of fuzzy multi criteria decision making methodologies for energy policy making. Energy Strategy Reviews, 24, 207-228.

Liu, C.; Le Roux, L.; Körner, C.; Tabaste, O.; Lacan, F.; Bigot, S. (2020) Digital Twin-enabled Collaborative Data Management for Metal Additive Manufacturing Systems. J. Manuf. Syst. S0278612520300741.

Miles, D., Marsden, W., \& Warde, S. (2016). Overview of additive manufacturing informatics:"a digital thread". Integrating Materials and Manufacturing Innovation, 5(1), 114-142

Müller, J. R., Siiskonen, M. D. I., \& Malmqvist, J. (2020). Lessons learned from the application of enhanced function-means modelling. In Proceedings of the Design Society: DESIGN Conference (Vol. 1, pp. 13251334). Cambridge University Press.

Naghshineh, B., \& Carvalho, H. (2020). The Impact of Additive Manufacturing on Supply Chain Resilience. In Doctoral Conference on Computing, Electrical and Industrial Systems (pp. 214-221). Springer, Cham.

Rayna, T., Striukova, L. (2016) From rapid prototyping to home fabrication: how 3D printing is changing business model innovation. Technol. Forecast. Soc. Change 102, 214-224

Rupal, B. S., Ahmad, R., \& Qureshi, A. J. (2018). Feature-based methodology for design of geometric benchmark test artifacts for additive manufacturing processes. Procedia Cirp, 70, 84-89.

Savolainen, J., \& Collan, M. (2020). How Additive Manufacturing Technology Changes Business Models?Review of Literature. Additive Manufacturing, 32, 101070. 